\title{
To Release or Not to Release: Evaluating Information Leaks in Aggregate Human-Genome Data
}

\author{
Xiaoyong Zhou, Bo Peng, Yong Fuga Li, Yangyi Chen, Haixu Tang, \\ and XiaoFeng Wang \\ Indiana University, Bloomington
}

\begin{abstract}
The rapid progress of human genome studies leads to a strong demand of aggregate human DNA data (e.g, allele frequencies, test statistics, etc.), whose public dissemination, however, has been impeded by privacy concerns. Prior research shows that it is possible to identify the presence of some participants in a study from such data, and in some cases, even fully recover their DNA sequences. A critical issue, therefore, becomes how to evaluate such a risk on individual data-sets and determine when they are safe to release. In this paper, we report our research that makes the first attempt to address this issue. We first identified the space of the aggregate-data-release problem, through examining common types of aggregate data and the typical threats they are facing. Then, we performed an in-depth study on different scenarios of attacks on different types of data, which sheds light on several fundamental questions in this problem domain. Particularly, we found that attacks on aggregate data are difficult in general, as the adversary often does not have enough information and needs to solve NP-complete or NPhard problems. On the other hand, we acknowledge that the attacks can succeed under some circumstances, particularly, when the solution space of the problem is small. Based upon such an understanding, we propose a risk-scale system and a methodology to determine when to release an aggregate data-set and when not to. We also used real human-genome data to verify our findings.
\end{abstract}

\section{Introduction}

With rapid advancement in genome sequencing technologies, human genomic data has been extensively collected and disseminated to facilitate human genome studies (HGS). A prominent example is genome-wide association study (GWAS) [4], a research technique that has been demonstrated to be highly valuable for identifying the genetic factors underlying common diseases. In a GWAS study, a group of participants with a disease/phenotype of interest (cases) are genotyped to compare the statistical features of their single-nucleotide polymorphisms (SNPs) 1 to those of the individuals without the disease/phenotype (controls). It is highly desired that the DNA data gathered during this process can be conveniently accessed by other researchers, which will greatly benefit the HGS community. Such data dissemination, however, needs to be balanced with the protection of participants' privacy, which is of paramount importance to this kind of research: for example, revealing the identity of a case individual in a GWAS relates her to the disease under the study, which can have serious consequences such as denial of access to health/life insurance, education, and employment. Prior research

${ }^{1}$ Common terminologies of genomics are summarized in Appendix 
shows that raw DNA data (genotypes) is often too risky to publish even after removal of explicit identifiers (such as name, social security number, etc.), as de-anonymization of a participant's identity can happen through examining the genetic markers related to her observable features (a.k.a. phenotypes) [8] 2 . What has been thought to be of low risk is aggregate genome data, such as allele frequencies, i.e., the frequencies of different SNP values, because such data covers an individual's information with that of others. As an example, the NHGRI/NIH used to make allele frequencies publicly available.

Aggregate Data Releases. A recent development in inference technologies, however, has completely changed the risk perception associated with the aggregate data. Particularly, Homer et al [39] discovered that the presence of an individual in a case group can be reliably determined from allele frequencies using the victim's DNA profile, which can be acquired, for example, from a single hair or a drop of blood. In response to this finding, the NIH swiftly removed all aggregate genome data from the public domain to protect the participants of HGS and avoid legal troubles [2]. Today, those who want to access the data have to file an application and sign an agreement, a complicated procedure that is time consuming. This becomes a hurdle to the dissemination of the data critical to HGS, and as a result, provokes intensive debates [10]: some researchers pointed out that the NIH may have overreacted, as the attack power achievable over at least some data-sets can be very limited [51,21]. On the other hand, such agreementbased protection has been found to be insufficient, as confidential user information can still be derived from other public sources: a recent study [52] shows that even the test statistics (e.g., p-values, r-squares) calculated from allele frequencies and published in HGS papers give away a significant amount of information, in some cases enough for identifying participants or even recovering portions of their DNA sequences. To make things worse, HGS researchers typically receive little guidance on what they are not supposed to share. Oftentimes, fine-grained allele frequencies/test statistics can be directly acquired from the authors of HGS papers.

Our Work. The current way aggregate human DNA data is handled indicates a disturbing lack of understanding of its privacy implication: such data have been both overprotected, which unnecessarily restricts their availability to the HGS researchers, and underprotected, which exposes the HGS participants to privacy threats. Crucial to the progress of the human genome research, therefore, becomes an in-depth study on how to evaluate the information leaks in the aggregate data and determine when they are safe to release, which also poses a challenge to the privacy researchers. This paper reports our research that makes a first step toward this end. We consider two types of common aggregate data, the allele frequencies for both individual SNPs and SNP pairs, and the test statistics derived from the frequencies. Such data is studied under two typical threats, identification attack that uses an individual's DNA profile to determine her relation with an aggregate data-set [39, 52, 46], and recovery attack that re-constructs individuals' SNP sequences from such data. Our paper investigated the feasibility of these attacks on different data based on information-theoretic and computational analyses. We further explored the potential to build a risk scale system.

\footnotetext{
${ }^{2}$ The NIH's guideline for sharing GWAS data [8] explicitly states "the NIH takes the position that technologies available within the public domain today, and technological advances expected over the next few years, make the identification of specific individuals from raw genotype-phenotype data feasible and increasingly straightforward".
} 
Contributions. We summarize the contributions of this paper as follows:

- Fundamental studies on information leaks in aggregate data. We performed both information-theoretic and complexity analyses on the common threats to different types of aggregate data. Our research sheds light on the fundamental questions on whether an attack on a specific data-set is feasible and how difficult it can be. Of particular importance here is our consideration of the special features of human genomes, which, as we show in the paper, can have significant impacts on the answers to these questions.

-Preliminary research on a risk-scale system. We propose a risk-scale system to classify aggregate data and guide the release of such data. Our research, though preliminary, is the first attempt to evaluate the risk of information leaks in a broad spectrum of aggregate data, including both single and pair-wise allele frequencies and different test statistics.

Roadmap. The rest of the paper has been organized as follows: Section 2 introduces background knowledge; Section 3 and 4 elaborate our research on the data release problems; Section 5 surveys the related research and Section 6 concludes the paper and discusses the future research.

\section{Backgrounds and Assumptions}

\subsection{Aggregate Human-Genome Data}

Our research has been conducted on two types of aggregate genomic data, allele frequencies and test statistics. Both are among the most valuable data to human genome research and are also most widely disseminated: for example, the former has been published by the NIH [7] and the latter are elaborated in every GWAS paper [53,47, 44, 25].

Each SNP has two alleles, encoded as 0 (major) or 1 (minor). Using this encoding scheme, the DNA profiles (containing the nucleotide sequences of the participants) of $N$ individuals $L$ SNPs, could be simply represented as a $N \times L$ matrix. Figure 1 gives an extremely small sample of encoded SNP profiles of 5 participants and 8 SNPs. The single-allele frequencies $f_{i}^{p}$ of a SNP site are the frequencies of the site's 'alleles, and the pair-wise allele frequencies $f_{i j}^{p q}$ of a SNP pair represent the frequencies of site $i$ and $j$ of the four allele combinations: $p q \in\{00,01,10,11\}$. Note that allele frequencies can be simply calculated from allele counts by dividing $N\left(\right.$ e.g. $\left.f_{i j}^{p q}=C_{i j}^{p q} / N\right)$.

From the allele frequencies, test statistics are often computed in different humangenome studies. Particularly, GWAS researchers utilize association tests to detect the SNPs related to the disease under the study. These tests compare the single-allele frequencies of the case population with those of the control population, in the hope of

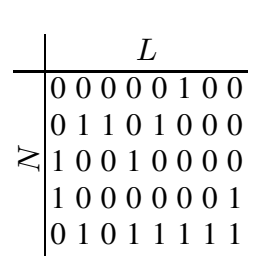

Fig. 1. A $0-1$ encoded SNP profiles of $N=5$ individuals and $L=8$ SNPs

\begin{tabular}{l|l|l} 
Data & Name & Sample \\
\hline$C_{i}$ & single allele count for SNP $i$ (major) & $C_{1}=3, C_{3}=4$ \\
\hline$C_{i j}^{p q}$ & pair wise allele counts for SNP $i$ and $j$ & $C_{12}^{10}=2, C_{13}^{\text {OO }}=2$ \\
\hline$C_{i j}^{p *}$ & single allele count for SNP $i$ & $C_{12}^{1 *}=2$ \\
\hline$r_{i j}^{2}$ & $r$-square, measures association and LD & $\frac{\left(C_{i j}^{00} C_{i j}^{11}-C_{i j}^{01} C_{i j}^{10}\right)^{2}}{C_{i j}^{0 *} C_{i j}^{1 *} C_{i j}^{* 0} C_{i j}^{* * 1}}$
\end{tabular}

Fig. 2. Routinely published data (single allele counts without superscript means major counts, e.g. $C_{i}=C_{i}^{0}$ ) 
identifying the genetic marker of the disease. The significance of each SNP (i.e., the strength of its tie to the disease) is measured by a p-value. Typically, those with pvalues below $10^{-7}$ are selected as putative markers. Such marker-disease associations can also be quantified using other test statistics such as odds ratios.

In addition to analyzing individual SNPs, a GWAS also examines the putative marker's associations with other SNPs in the same genetic locus, called linkage disequilibrium (LD) [45], which could also have a connection with the disease. LD of a locus is typically measured by the test statistics such as D' and r-square, which are calculated from pairwise allele frequencies of the locus. Sometimes, researchers further analyzed the allele combinations involving multiple correlated SNPs, i.e., haplotypes, which are inferred from genotypes through a class of phasing algorithms [1, 50, 49].

Figure 2 shows how to calculate these test statistics and some sample values for Figure 1, which are routinely published in HGS papers [28, 48, 47, 53]. Oftentimes, these papers include the p-values of hundreds of SNPs and figures that illustrate their LDs. More detailed information can also be acquired from the authors. In our research, we focused on p-values and r-squares, the two most-commonly reported test statistics.

\subsection{Threats}

The threats studied in our research include identification attack and recovery attack, two major privacy concerns in human genome research. The first identification attack on aggregate data has been proposed by Homer, et al [39], which requires availability of a SNP profile from the victim. The objective here is to determine the presence of an individual in the case group, so as to relate her to a disease. To this end, the attacker runs a statistic test that evaluates whether the victim's SNP profile is independent from the single-allele frequencies of the case population. Let $Y_{j} \in\{0,1\}$ be the allele of SNP $j$ in the profile, and $\hat{f}_{j}^{0}$ and $f_{i}^{0}$ be the major allele frequencies of that SNP in the case population and a reference population, respectively. Homer's attack measures the following distance:

$$
D\left(Y_{j}\right)=\left|Y_{j}-f_{j}^{0}\right|-\left|Y_{j}-\hat{f}_{j}^{0}\right|
$$

Under the assumption that the distributions of individual allele frequencies are identical in the case and the reference, the sum of $D\left(Y_{j}\right)$ across a large number of SNPs follows a normal distribution with a zero mean if the victim is not present in the case group. Otherwise, the sum becomes positive and significantly deviates from the mean. In their paper, the authors report identification of a case individual with a extremely low false positive rate, given 25,000 SNPs of the victim. This line of research has been followed by multiple research groups [46, 21, 51, 40, 52]. Particularly, Sankararaman, et al [46] utilized the likelihood ratio test to estimate the upper-bound of the identification power achievable on single-allele frequencies. They also built a tool called SecureGenome [11] to evaluate such a threat on different data sets.

Besides single-allele frequencies, pair-wise allele frequencies and test statistics were also found to leak out a substantial amount of information. In prior research [52] , it was found that the identification attack can happen to even the test statistics published in GWAS papers, through a statistical test based upon signed $\mathrm{r}$ values. Given $N$ sequences of $L$ neighboring SNPs in the genome, the signed $r_{i j}$ between two SNPs $i$ and $j(1 \leq$ $i<j \leq L)$ is defined as $r_{i j}=\frac{C^{11} C^{00}-C^{01} C^{10}}{\sqrt{C^{1 *} C^{0 *} C^{* 1} C^{* 0}}}$, where $C^{p q}$ is the pair-wise allele 
counts, i.e. the number of the sequences with allele $p(p \in\{0,1\})$ at SNP $i$ and allele $q$ $\left(q \in\{0,1\}\right.$ ) at SNP $j$, and $C^{p *}$ and $C^{* q}$ are single allele counts. $r_{i j}$ can be computed from $r_{i j}^{2}$ (Figure 2) except its sign. Like Homer's approach, the attack needs a reference population whose $\mathrm{r}$ values are denoted by $r^{R}$, in addition to the case population $\left(r^{C}\right)$, and a SNP profile from the victim in which $Y_{i j}^{p q} \in\{0,1\}$ indicates whether her SNP pair $i j$ has a pair-wise allele $p q$. A test statistic $T_{r}$ is thus constructed as follows:

$$
T_{r}=\sum_{1 \leq i<j \leq N}\left(r_{i j}^{C}-r_{i j}^{R}\right) \cdot\left(Y_{i j}^{00}+Y_{i j}^{11}-Y_{i j}^{01}-Y_{i j}^{10}\right)
$$

$T_{r}$ is much more powerful than the statistical attacks on single allele frequencies [52], as it makes use of the relations among SNPs, the linkage disequilibrium, which contain much more information than individual SNPs. A problem here, however, is the need to know the signs, which is not typically released. They are determined in the prior work [52] by taking advantage of integer constraints, base upon the assumption that the published r-squares are calculated from allele counts (integers) and are not perturbed by noise.

The recovery attack aims at re-constructing the SNP sequences (i.e., haplotypes) used in an HGS: prior research [52] reports a successful restoration of 100 sequences involving 174 SNPs on a locus from their single and pair-wise allele frequencies. Note that these frequencies can be estimated through reverse engineering the test statistics published in GWAS papers [52]. Compared with the identification attack, such an attack can be more difficult to succeed and consume much more computing resources. However, it does not rely on the DNA profile from the victim.

An ideal privacy goal here is differential privacy [29], which ensures that two aggregated datasets differing from each other by one individual's data have indistinguishable statistical features. An example when this happens is that the data from a very large number of participants is aggregated so that the contribution of an individual becomes negligible. This privacy goal, once achieved, can defeat inference attacks using all kinds of background knowledge. However, this condition is known to be hard to satisfy in a practical system. For genomic data, the knowledge about the victim's DNA profile and a good reference population is deemed as a strong assumption in the adversary's favor [51,21]. Based on such an assumption, we thoroughly studied the feasibility and complexity of these two types of attacks on the two types of datasets, and the methodology to determine whether a specific set of data is safe to release. Due to the space limit, this paper focuses on two most interesting scenarios where allele frequencies face the recovery attacks and test statistics are under the identification threat. The other two cases, i.e., the identification threat to allele frequencies and the recovery threat to test statistics, are much simpler: for example, the former has already been preliminarily explored by the prior research [11]. Our new findings on these cases can be found in a longer version of the paper [55].

\subsection{Adversary Model}

We consider a probabilistic polynomial time adversary who can not accomplish the task that needs exponential computing power, for instance, sampling an exponential space to determine a probability distribution over this space. Other than that, we assume the adversary has sufficient resources and perfect information at her disposal for individual attacks. Specifically, for the identification attack, we consider that the adversary 
has access to the victim's DNA profile and a good reference population with an allele distribution identical to that of the case population. This is the best resource such an attack can expect [52,39]. For the attack involving test statistics, we assume that highprecision data is available, which affects the outcome of such an attack, as indicated in the prior research [52].

\section{Recovery Threats to Allele Frequencies}

Given a set of pairwise allele frequencies, a recovery attack aims at partially recovering the haplotype sequences of HGS participants, which is completely realistic according to prior research [52]. These sequences, once restored, can be used to re-identify these participants, a threat well recognized by the NIH (see Footnote 1 and [8]). This section reports a new methodology for determining the susceptibility of different allele-frequency data to such an attack.

\subsection{The Problem}

Figure 3 illustrates the recovery attack, in which the adversary attempts to recover a matrix, with each of its row vectors being a haplotype sequence, from the constraints of pairwise allele frequencies 3 . This problem can be formulated as a haplotype matrix recovery problem below:

Haplotype Matrix Recovery Problem. Consider an $N \times L$ haplotype matrix $M$ that represents $N$ haplotype sequences over $L$ SNP sites. The set of pairwise allele frequencies of $M$ is denoted by $d=\left\{f_{i j}^{p q}\right\}$, where $p$ and $q$ are the allele types at SNP sites $i$ and $j$, respectively. Note that there are in total $\left(\begin{array}{c}L \\ 2\end{array}\right)$ such pairs among $L$ SNPs. Let $S$ be the space of $M$ (the matrix), and $D$ be the space of $d$ (the pairwise allele frequency). Given $d$ and $N$, the adversary intends to recover the haplotype matrix, that is, to find an $M^{\prime}$ in $S$, which is equivalent to $M$ ignoring the order of their row vectors.

It is conceivable that in some cases (some pairwise allele frequency $d$ ) the problem has unique solution: that is, there exists a unique matrix $M$, disregarding the ordering of its rows, that satisfies the constraints imposed by $d$, whereas in some other cases, the problem has no solution (i.e. the pairwise allele frequencies are not satisfiable), and in the remaining cases, the problem has multiple solutions. Figure 4 illustrates an example that multiple solutions exists for a given $d$. If there are multiple solutions and the intersection of all the solutions is small, when an attacker gets one solution, she has low confidence if any of the sequence in his solution is indeed in the original haplotype matrix.

Challenges in Risk Classification. To determine the risk scale of a given frequency set $d$, we first find out whether it has multiple solutions. If this is true and the overlap among these solutions is sufficiently small, we can comfortably put the set in the Green zone. Unfortunately, this decision turns out to be extremely difficult to make, because several problems on the haplotype matrix recovery are computationally hard. Specifically, we found that:

Theorem 1. Determining if there is a haplotype matrix for a given pairwise allele frequency set is NP-complete.

\footnotetext{
${ }^{3}$ Note that the pairwise allele frequencies contain all the information of single allele frequencies.
} 


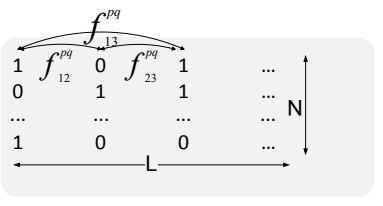

Fig. 3. Recovering a matrix from pairwise allele frequencies. Given a pairwise allele frequency set $d=\left\{f_{i j}^{p q}\right\}$, the attacker tries to recover the matrix satisfying $d$.

\begin{tabular}{lll|lll}
\hline 0 & 0 & 0 & 0 & 0 & 1 \\
0 & 1 & 1 & 0 & 1 & 0 \\
1 & 0 & 1 & 1 & 0 & 0 \\
1 & 1 & 0 & 1 & 1 & 1 \\
\hline
\end{tabular}

Fig. 4. The left matrix and the right matrix have exactly the same single allele frequencies and pairwise allele frequencies, but do not share any single haplotype sequence

Corollary 1. Determining the number of haplotype matrices for a given pairwise allele frequency set is NP-hard.

Conjecture 1. Determining if a solution is unique for a given pairwise frequency set is Co-NP-complete.

Corollary 2. Recovering one haplotype matrix for a given pairwise allele frequency set is NP-hard.

Corollary 3. Determining if there exists a solution for a given pairwise allele frequency set that does not contain a given row vector is NP-complete.

Corollary 4. Recovering one haplotype matrix for a given pairwise allele frequency set that does not contain a given row vector is NP-hard.

Proofs are provided in Appendix B] Theorem 1 to Corollary 4 show that determining the existence of unique or multiple solutions for a given allele frequency set and recovering even single one of them are all hard problems. Note that proving average-case complexity is well known to be difficult [34]. Nevertheless, our empirical study using IBM Cplex [5] with parallel enabled suggests that at least the decision problems here do not seem to be easy in the average time. We randomly sampled 10 matrices of size $100 \times 80$ and put them on a workstation with 4 Quad-Core Xeon $2.93 \mathrm{GHz}$ processors, none of them could be solved within one week.

Determination of Risk Scales. In spite of the difficulty in finding the number of solutions, it is still plausible to estimate whether a given frequency set is likely to have multiple haplotype matrix solutions, by considering solely the size of the recovery problem as determined by two parameters, i.e., the number of SNP sites $L$ and the number of haplotype sequences $N$. We compare the solution space $\|S\|$ and the frequency set space $\|D\|$. When $\|D\| \approx\|S\|$, the corresponding frequency set is likely to have a unique haplotype matrix solution. Conversely, when $\|S\| \gg\|D\|$, a data-set $d$ becomes very likely to have multiple solutions. Intuitively, the distribution of the solutions over the different $d$ tends to have a very small deviation: that is, it is unlikely that only a few have many solutions while the others have unique ones. Furthermore, because the distribution is over a large number of variables (i.e. the elements in the haplotype matrix) and it is very complicated, the adversary cannot estimate the distribution without using exponential computing power. The adversary, who is unsure about the uniqueness of the solution, but, on the other hand, is aware of the strong indications that multiple 


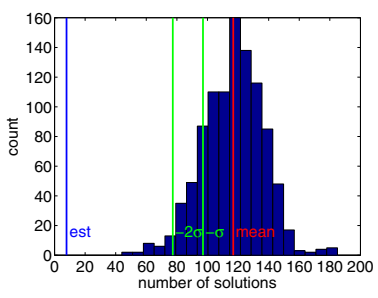

Fig. 5. Solution Distribution. $(\mathrm{N}=40, \mathrm{~L}$ $=7$, sample size $=1000$, space ratio (estimated number of solutions) $=7.861$, average $=116.855)$

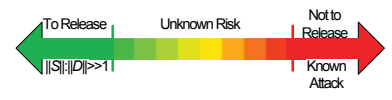

Fig. 6. Risk spectrum. When $\|S\|$ : $\|D\| \gg 1$, data is placed in the Green zone. If there is a known attack, data must be placed in the Red zone. Otherwise further investigation is needed for the data (Yellow Zone).

solutions exist, will end up with little faith in any solution she is able to find. What is more, she may not even know how close to the real haplotype sequences her solution is, if $\|S\|$ becomes sufficiently large to ensure that many data-sets have multiple solutions.

Although it is difficult to rigorously define the distribution of solutions over $d$, we conducted an empirical study to verify our hypothesis that the solutions distribute near randomly. We randomly sampled 1000 haplotype matrices of size $N=40$ and $L=7$, and calculated their pairwise allele frequencies. Using each set of these pairwise allele frequencies $d$ as constraints, we computed for each instance all solutions that can be found by Cplex, a state-of-the-art NP solver [5] 5. As expected, the distribution of the number of solutions is close to a normal distribution with a small standard deviation (Figure 5). The standard deviation (19) is on the same scale as the square root of the mean (116), indicating that it is unlikely that only a few $d$ have many solutions while others have only a few or single solutions.

The above analysis indicates that we can have a shade-of-grey risk spectrum, as illustrated in Figure 6, which is approaching the Green end with the increase of the ratio $\|S\|:\|D\|$. Intuitively, this suggests that the larger the ratio, the less the adversary knows about the distance between her solution and the real one 6 . Upon the spectrum, we can use a distance threshold to determine when a frequency data-set can be designated to the Green zone. This research is elaborated in Section 3.2 and 3.3

Towards the Red end of the spectrum, we proved that restoring a solution matrix from allele frequencies is NP-hard, even if the solution is known to be unique. However, we also acknowledge that the special features of human-genome data, particularly the LD relations among them, could make the problem tractable, as indicated in prior research [52]. Therefore, a conservative approach is to label a data-set "Red" only when

\footnotetext{
${ }^{4}$ We chose this problem scale because $L$ and $N$ met condition 3 which we will discuss shortly and the problem is small enough to be solved by Cplex in reasonable time.

${ }^{5}$ We did not enumerate all putative solutions. Instead, we set the populate limit of Cplex as 200 to save memory and time. Hence, the number of solutions shown here may be smaller than the actual number of solutions.

${ }^{6}$ An exception here is some special cases, for example, when the frequencies of the pairwise allele type 00 become 1 for all SNP pairs. Such cases, however, can be identified before the data being released.
} 
it is found to be vulnerable to a known attack. Otherwise, the data-set is put in the Yellow zone, awaiting further investigation, if it is also not qualified for the Green zone. The details of this analysis are presented in Section 3.4

\subsection{When to Release}

As discussed above, when the solution space becomes sufficiently larger than the space of allele-frequency sets, the threat of recovery attack can be mitigated, as the adversary cannot determine whether a given frequency data-set describes a unique set of SNP sequences. Here, we present an analysis on how large the solution space needs to be.

Solution-Space Analysis. Let us first consider the solution space $S$. For $L$ SNPs, there are $2^{L}$ possible SNP sequences. The number of different solutions, each of which is an $N$ by $L$ haplotype matrix, is at least $\left(\begin{array}{c}2^{L} \\ N\end{array}\right)$, i.e., selecting $N$ distinctive sequences from the $2^{L}$ sequences.

Then, we estimate the space of pairwise allele frequency sets $D$. Given $N$ and a frequency set $d=\left\{f_{i j}^{p q}\right\}$, we can have a set of pairwise allele counts $\left\{C_{i j}^{p q}\right\}$, which directly determine the set of single allele counts $\left\{C_{i}\right\}$. Since for any SNP pair, the frequencies of one pairwise allele and one single allele are sufficient for inferring the frequencies of other alleles, pairwise or single, for the same SNP pair (see Inequality 3 in [52]), the set $d$ is uniquely determined by $\left\{C_{i}\right\}$ and the set of pairwise major allele counts, which we denote by $\left\{C_{i j}\right\}$ for simplicity.

From the fact that $C_{i j}$ and $C_{i}$ can take any value in $[0, N]$ and there are $\left(\begin{array}{l}L \\ 2\end{array}\right)$ SNP pairs and $L$ single SNPs, we know that the number of different frequency sets $d$ will not exceed $(N+1)^{\left(\begin{array}{l}L \\ 2\end{array}\right)} \cdot(N+1)^{L}=(N+1)^{\left(\begin{array}{l}L \\ 2\end{array}\right)+L}$. Comparing $\|S\|$ with $\|D\|$, we can get a necessary condition for the existence of multiple solutions: $\left(\begin{array}{c}2^{L} \\ N\end{array}\right)>(N+1)^{\left(\begin{array}{c}L \\ 2\end{array}\right)+L}$. But it is too complex to use. Using Stirling's approximation, we get $\frac{2 N L}{L(L-1)+2 L}\left(1-\frac{\log \frac{N}{e}}{L}-\right.$ $\left.\frac{\log 2 \pi N}{2 N L}\right)>\log (N+1) 7$. This gives us $\frac{2 N}{L+1}\left(1-\frac{\log \frac{N}{e}}{L}-\frac{\log 2 \pi N}{2 N L}\right)>\log (N+1)$. For $L>200,1-\frac{\log \frac{N}{e}}{L}-\frac{\log 2 \pi N}{2 N L} \approx 1$. Ignoring other constants, we get the following condition:

$$
\frac{2 N}{\log (N+1)}>L
$$

Partial Recovery of Haplotype Matrix. The above analysis did not take into consideration the possibility that multiple solutions, although they exist, are close enough to each other for a given set of pairwise allele frequencies, e.g., there are a significant number of sequences shared between them. If this occurs and the attacker somehow recovered all the solutions (even though it is NP-hard, Corollary 1), and makes an intersection over these solutions, she knows the resulting common sequences must be in the case group. To defend against such attacks, we need stronger condition to assure the security of the pairwise allele frequency data to be released: for a specific haplotype sequence, there should exist another haplotype matrix solution that does not contain this sequence. When this happens, even if an attacker manages to obtain a solution (i.e. a set of haplotype sequences), she is not confident that any sequence in her solution is present in the actual haplotype matrix, because for any such sequence, there is always

\footnotetext{
${ }^{7}$ Unless otherwise specified, $\log$ means $\log _{2}$ in this paper.
} 
another haplotype matrix that is equally likely to be the actual matrix and also does not contain this sequence (although to find this matrix is NP-hard according to Corollary 4). Similarly, even if the attacker obtained multiple solutions, the intersection of these solutions will not give her any confidence that the sequence in the intersection must be present in the actual matrix.

To get this stronger condition, we consider the solution space for a given instance $d$ with $N$ rows (sequences) and $L$ columns (SNP sites), but one haplotype sequence in the original matrix is not in these solutions. This is equivalent to the entire matrix space, i.e., $\frac{2^{N \times L}}{N !}$, subtracted by the matrix space with one fewer row (set as the given haplotype sequence), i.e., $\frac{2^{(N-1) \times L}}{(N-1) !}$. By using the same analysis from above, we get the following condition:

$$
\frac{2(N-1)}{\log (N+1)}>L
$$

Once the size of a haplotype matrix ( $N$ and $L)$ meets this condition, its solution space will become sufficiently large that the intersection of all of its solutions is unlikely to contain even one haplotype sequence. This condition is also very close to that of Condition 3 .

Empirical Study. To verify whether the above privacy assurance is sufficient in practice, we conducted an empirical study on a number of small-scale problems. We randomly sampled 30 haplotype matrices that satisfy the condition (with $N=40$ and $L=8$ ), and for each haplotype sequence in the original matrix, we attempted to recover another haplotype matrix that did not contain this sequence but still has the same pairwise allele frequencies as those of the original matrix. Again, we used Cplex to search for all matrix solutions (with a populate limit of 200). In the end, for each of the haplotype sequences in the 30 matrices we sampled, at least 74 solutions were found that did not contain that sequence, indicating that given any haplotype sequence in a matrix, there likely exists an alternative solution (another haplotype matrix) associated with the pairwise frequency set of the original matrix, which does not include that sequence. This study shows that condition 4 can be used to estimate when a pairwise frequency set is unlikely to be vulnerable to an intersection attack.

\subsection{The Impact of Human Genetic Structure}

A critical pitfall in the analysis above is that it does not take into consideration the prominent features of human genome sequences. Instead of being random binary sequences ( 0 for major and 1 for minor allele) as assumed in our model, human genome sequences contain complex structures that are well studied in human genetics and can be inferred from publicly accessible human genome data [13,6]. Thus, the adversary could simply examine a solution she finds to determine whether it looks like a human genome sequence. This leads to the further reduction of the solution space $\|S\|$. In this section, we present another analysis based upon a human genetic model.

Human Genetic Model. We model haplotype sequences with a Markov chain (MC), a standard approach extensively used in human genetic research for the modeling of the LD structure (single and pairwise allele frequencies) in a specific genetic locus [35, 42, 43]. Given $L$ SNP sites, the model can be represented as a heterogeneous 


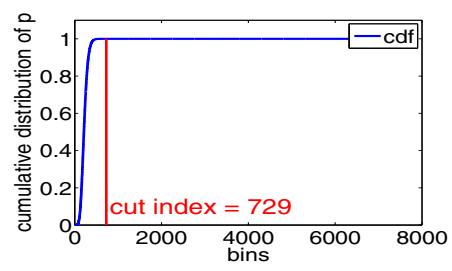

(a) cdf

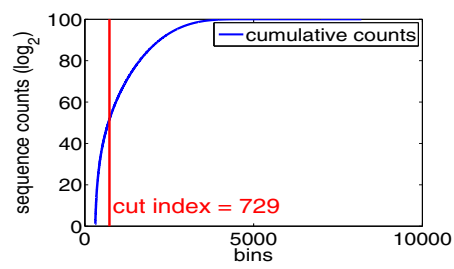

(b) counts

Fig. 7. The Markov Chain model for estimating the effective solution space. (a) Cumulative distributions of the probabilities of haplotype sequences, sorted in descending order of probabilities. Cutoff probability $\theta=0.99999$. (b) Total number of most probable sequences vs. their cumulative probabilities. Vertical red lines represent the cutoff.

Markov chain with a sequence of $L$ states $\left(X_{1} X_{2} \ldots X_{L}\right)$, where $X_{i} \in 0,1$, representing the major (0) or minor (1) allele, and an initial probability distribution (denoted by $P^{0}\left(X_{1}\right)$ ) as well as $L-1$ different transition probability matrices (denoted by $\left.P^{i}\left(X_{i+1} \mid X_{i}\right)\right)$ are used to model the transition probabilities from the $i$-th state to the $(i+1)$-th state, which are estimated from the single and pairwise allele frequencies using standard methods [35, 42, 43]. As a result, each of the $2^{L}$ haplotype sequences corresponds to a state sequence and the probability of observing it under the MC model can be computed by $P\left(X_{1} X_{2} \ldots X_{L}\right)=P^{0}\left(X_{1}\right) \cdot \prod_{i=1}^{L-1} P^{i}\left(X_{i+1} \mid X_{i}\right)$. Once built from a group of haplotype sequences from human individuals (e.g. the case group or a reference group), the MC model can be used to evaluate the effective space of haplotype matrices that are likely sampled from real human individuals. Among totally $2^{L}$ possible haplotype sequences, the probabilities of observing some sequences are so low that they are deemed unlikely to appear in human genomes, owing to the strong associations among neighboring SNPs. These sequences should not be considered when estimating the solution space of haplotype matrices. Assume the probabilities of $2^{L}$ sequences and a threshold $\theta$ (close to 1, e.g. 0.99999) are given, the effective space of haplotype sequences can be estimated by the number of most probable sequences that have a cumulative probability greater than $\theta$. This was achieved in our research through an approximation algorithm which is given in our technique report [55].

Evaluation. To estimate the solution space under a human genetic model, we phased 3008 sequences from WTCCC ch7 of 100 SNPs by using PHASE [3]. We chose $2 T=$ 8192 bins to estimate distribution of haplotype sequences under the MC model. As shown In Figure 7(a), with cutoff probability $\theta=0.99999$, only 729 bins of $\approx 2^{52}$ most probable sequences are obtained, as compared to the entire space of $2^{100}$ haplotype sequences, which indicates that the incorporation of the human genetic model significantly reduces the effective space of haplotype sequences. Figure 8 shows the space comparison between $\|S\|$ and $\|D\|$. We could see that in the original analysis, we need about $5 L$ sequences to ensure multiple solutions for the given pairwise allele frequencies. Defending against the intersection attack requires pretty much the same number of sequences as shown in the figure. To incorporate the human genetic model (the MC model), we need roughly $12 L$ sequences. 


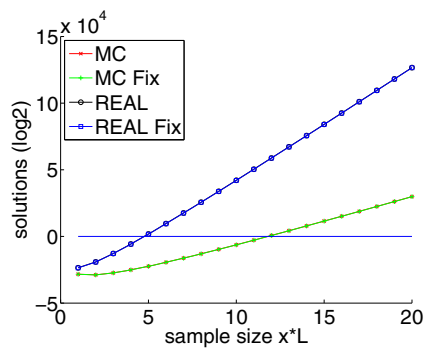

Fig. 8. Comparison between matrix space $\|S\|$ and constrain space $\|D\|$ for data fromWTCCC1 of SNP 100

\subsection{When Not to Release}

For the frequency set that cannot be put in the Green zone, its solution is likely to be unique. The adversary who finds the solution has reason to believe that it is the correct one. Here, we elaborate how to classify such a data-set.

Red-Zone Data. Although recovering SNPs sequences is NP-hard in general, the special features of human genome can enable the attack to succeed on at least some frequency sets. Prior research reports a successful attack on a data-set related to $100 \mathrm{SNP}$ sequences and 174 SNPs from the FGFR2 locus [52]. The approach leverages the LD relations among these SNPs to break the matrix into small blocks in a way that preserves the strong inter-SNP relations within individual blocks. Such relations allow the adversary to first restore individual blocks and then use the aggregated relations between blocks to connect them together.

To avoid releasing the data vulnerable to the recovery attack as well as overprotecting those that can actually be disclosed, we suggest to test a frequency set with known attacks and assign it to the Red zone when it is exploited. If the attacks fail, we can label the data-set as "Yellow" to leave the decision on its release to the future research.

\section{Identification Threat to Test Statistics}

Besides allele frequencies, also widely disseminated by HGS are the test statistics computed from these frequencies. Particularly, HGS papers routinely report p-values and rsquares $\left(r^{2}\right)$ over tens or even hundreds of SNP sites. Prior research [52] shows the key to an identification attack on such data is knowledge of the values of $r$ or equivalently, their signs (given $r^{2}$ ). Once such information is given, we can use $T_{r}$ [52] to decide whether a set of r-squares can be released, in the same way as SecureGenome [11] does to single and pairwise allele frequencies. Specifically, we can release such a data-set if given all correct signs, the achievable statistical power on it, as reported by $T_{r}$, is still below a threshold. However, when the power turns out to be high, a decision to keep the data off limit can be premature: after all, there we assume that all the signs are recovered, which is by no means easy in practice, as discussed later in this section. Therefore, a question becomes how to seek a "tighter bound", allowing the statistics to be released when it is too difficult to recover a dangerous amount of information from them. This issue is addressed in this section. 
The rest of the section presents our understanding of the problem: how sign recovery improves the chance of successful identifications and how difficult this can be done. Then, we come up with the yardsticks for releasing test statistics and describe a new potent attack that helps decide when data should be held from publication.

\subsection{The Problem}

An important question we are asking is how many correct signs a successful attack needs. The answer sheds light on the conditions under which the attack becomes ineffective. To find out the answer, we can analyze the relations between the rate of the correct signs used in an optimal test and the statistical power it can achieve on a particular data-set. Specifically, given a rate of correct signs $\alpha$, we can randomly assign correct signs to the $r$ of a fraction $\alpha$ of SNP pairs, and then run $T_{r}$ under the assignment to determine its power, i.e., the rate of successful identifications. This test needs to be conducted repeatedly for each rate of correct signs, to get the maximum power under different sign assignments. In this way, we can obtain an estimated power-sign relation, and then use a threshold to determine the maximum rate of correct signs that will not pose a serious identification threat.

Complexity of Releasing Statistics. Given a threshold $\alpha(\alpha \in[0,1])$ of the correct sign rate, a set of test statistics (r-squares) can be placed in the Green zone if the adversary cannot correctly recover as many as $\alpha$ of all $\left(\begin{array}{l}L \\ 2\end{array}\right)$ signs. This can be ensured if the set of $r$-squares is mapped to multiple sets of valid signed $r$ values, and the overlap among these sets is below the threshold $\alpha$. When this happens, the adversary, even if she can recover all these sets of signed $r$ values, cannot identify enough signs with any confidence for a successful attack. Obviously, given $\left(\begin{array}{c}L \\ 2\end{array}\right)$ r-squares over $L$ SNP sites, there are totally $2^{\left(\begin{array}{c}L \\ 2\end{array}\right)}$ possible sign assignments, with each of them corresponding to a different set of signed $r$ values. However, not all of such assignments are valid: many of them do not correspond to any haplotype matrix, as those assignments lead to the $r$ values inconsistent with each other.

We studied a sign recovery problem: given a set of r-square values $r_{i j}^{2}$ over $L$ SNP sites, a set of single allele frequencies $f_{i}(i=1,2, \ldots, L)$, which could be recovered from p-values [52], and the total number of sequences in the case group $(N)$, find a set of signed r values $\hat{r}_{i j}$ so that (1) $r_{i j}^{2}=\hat{r}_{i j}^{2}$; and (2) $\hat{r}_{i j}$ are valid, i.e. there exists a haplotype matrix whose pairwise allele counts $C_{i j}^{p q}(p, q \in 0,1)$ satisfy $N \cdot f_{i}=\sum_{q \in\{0,1\}} C_{i j}^{0 q}$ for all $i$ and $j$, and $r_{i j}=\frac{C_{i j}^{00} C_{i j}^{11}-C_{i j}^{01} C_{i j}^{10}}{C_{i}^{0} C_{i}^{1} C_{j}^{0} C_{j}^{1}}$. Similar to the haplotype matrix recovery problem, several key problems related to the sign recovery problem are computationally hard if we assume the haplotype matrix has more than just a few rows (haplotype sequences). This can be satisfied by all real HGS studies, which typically contains hundreds of individuals. Specifically, under this condition, we show that:

Theorem 2. Determining if there exists a set of sign assignments of $r$ for a given set of $r$-squares and single allele frequencies is NP-complete.

Corollary 5. Recovering a valid sign assignment for a given set of $r$-squares and single allele frequencies is NP-hard.

Corollary 6. Finding the number of valid sign assignment for a given set of $r$-squares and single allele frequencies is NP-hard. 
The proofs are provided in technique report [55]. We note that these results have strong implications on classifying an r-square set into Green or Red zones. Briefly, an adversary faces the following computational difficulty: assume that she manages to recover some sets of signs from r-squares, which itself is NP-hard; she still has no clue whether there are any other valid sign assignment and how many correct signs have been discovered in her solution. In other words, she will not have any reasonable confidence in the identification she makes from the r-square data-set. There is an exception, though: if the solution space of valid sign assignments (or equivalently their corresponding haplotype matrices) is sufficiently small, for example, as small as the space of r-squares, then the adversary has a good reason to believe that every set of r-squares has a unique valid sign assignment. Here the situation is analogous with that in Case 2 (Section 3). Similarly, we need a solution-space analysis to ensure that the adversary cannot get any useful information from a data-set to be released.

\subsection{When to Release}

Before placing a data-set to the Green zone, we need to ensure that the adversary cannot recover enough signs from it to achieve any significant identification power. Consider that a polynomial-time adversary learns from the ratio between the space of r-squares $\left\|R^{2}\right\|$ and the space of matrices $\|S\|$ that an r-square set can have $\kappa$ solutions. Given a specific set of r-squares, she has no reason to believe that the set has fewer solutions, because she can neither determine the exact number of solutions nor sample the exponential space $S$ (when $N$ and $L$ are large) to estimate the distribution of matrices over r-square sets. Also, recovering all these matrices is NP-hard and therefore the adversary has no clue how many different sets of valid signs exist, except that the number will not exceed $\kappa$. When $\kappa$ is sufficiently large, the adversary, even after she manages to get a set of signs, does not know whether the overlap among all sets (which can be as many as $\kappa$ ) goes above $1-\alpha$ of all $\left(\begin{array}{l}L \\ 2\end{array}\right)$ signs.

Solution-Space Analysis. Therefore, the condition for the release of an r-square set is that $\|S\|:\left\|R^{2}\right\|$ should be sufficiently large to ensure that the adversary does not know whether she recovers enough correct signs. As described in Section $3\|S\| \approx$ $2^{L N}\left(\frac{N}{e}\right)^{-N}(2 \pi N)^{-1 / 2}$. Since the space size of the $\mathrm{r}$ values is approximately $(N+$ $1)^{\left(\begin{array}{l}L \\ 2\end{array}\right)+L}$, from $\mathrm{r}$ to r-squares, the space shrinks to $\left\|R^{2}\right\| \approx \frac{(N+1)^{\left(\frac{L}{2}\right)+L}}{2^{\left(\frac{L}{2}\right)}}$. To ensure multiple solutions, we need $\|S\|>\left\|R^{2}\right\|$, which gives:

$$
\frac{2 N}{\log (N+1)-1}>L
$$

For example, for a locus involving 100 SNPs, at least 225 individuals (450 haplotype sequences) should be in the case group to ensure the existence of multiple solutions. Not surprisingly, this is less stringent than the condition of placing a set of pairwise allele frequency in the Green zone (where one needs to have at least 500 sequences for a 100-SNP locus), because r-squares contain less information than the pairwise allele frequencies. To further prevent the adversary from identifying more than $1-\alpha$ of the correct signs, we need to make it possible to have an element in $R^{2}$ be mapped by 


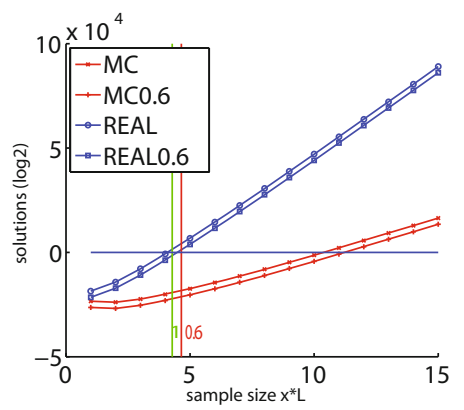

Fig. 9. Comparison between matrix space $\|S\|$ and $\left\|R^{2}\right\|$ for data from WTCCC1 of $L=100$ SNP. Vertical line shows the required sample size estimated from formula 5 and 6 and then added by a buffer of $0.5 \mathrm{~L}$.

at least $2^{(1-\alpha)\left(\begin{array}{l}L \\ 2\end{array}\right)}$ element 8 in $S$. To ensure this, we must have that $\|S\|$ is at least $2^{(1-\alpha)\left(\begin{array}{c}L \\ 2\end{array}\right)}$ times as large as $\left\|R^{2}\right\|$. This ultimately gives us the following condition:

$$
\frac{2 N}{\log (N+1)-1+\alpha}>L
$$

Considering Human Models. Again, when the special properties of human genomes are being considered, we need to re-assess the matrix space $\|S\|$ based upon a human genetic model, as described in Section 3.3. In our research, we ran the approximation algorithm (Section 3.3) to identify $L$ and $N$ that satisfy the above conditions (multiple sets of signs with a large distance), using the WTCCC1 data.

Figure 9 shows the result of the experiment involving 100 SNPs. As we can observe from the figure, in absence of a human model, a population with more than 250 individuals (500 sequences) are required to make sure that no more than $60 \%$ of signs can be identified. If we consider the human features, we need a population of at least 600 individuals $(N>1200)$.

\subsection{When Not to Release}

When the space of matrices $S$ comes close to that of the r-squares, the adversary knows that once she acquires a set of valid $r$ values, they are likely to be correct. Although we have shown that recovering signed $r$ values from $r$-squares is NP-hard (Corollary 5), some instances of the sign recovery problem may be easy to solve, in particular when a human genetics model is employed to help solve the problem. Here we present a new attack technique that helps determine when this situation occurs, and thus a dataset should not be released. The new attack leverage on the LD structure of human genome and using haplotype recombination to efficiently recover the sign. For more detail, please read our technique report [55].

Evaluations. We ported the LD function, which is used in many GWAS papers for calculating MLE $r^{2}$, from the snp.plotter [12] package of $\mathrm{R}$ [9] to Matlab and implemented

\footnotetext{
${ }^{8}$ Note that the adversary has to consider the situation that all these elements (matrices) are associated with different $r$ sets, as she has no computing power to estimate the relations between $r$ and matrices.
} 
the recombination attack using a stochastic hill climbing algorithm with multiple starting points. Then, we evaluated the attack on the data extracted from WTCCC1. We extracted 180 SNPs from chromosome 7. A case group and a reference group of 100 each were randomly sampled from the data-set. After that, the MLE-estimated $r^{2}$, together with single allele frequencies, was used as the optimization target for both inner block and inter block recombinations. On average, the sign agreement rate between the initial haplotype matrix (reference) and the target matrix (case) was $58.7 \%$, which had very small power (identification rate $3.0 \%$ under a false positive rate $1 \%$ ). After learning, the sign rate agreement was improved to $67.2 \%$ on average and the identification rate became $8.1 \%$ : that is, our approach enabled an adversary to identify about 8 participants from the aggregate data with a poor quality.

\section{Related Work}

The problem of releasing aggregate data while preserving their privacy has been extensively studied in privacy preserving data analysis [29,33], statistical disclosure control [18, 19, 32], inference control [24] and privacy-preserving data mining [14, 15]. However, the properties of human genome data make the problem special in this domain, which has not been well investigated. Especially, human individuals share about 99.9\% genomic sequences, which makes it easy to find a reference group from public sources such as HapMap [6]. This enables both Homer's attack and the statistical attack proposed in [52], as elaborated in Section 2.2. Also remotely related to our research is the work on privacy preserving genome computing [16,41,22], which however does not focus on protecting the outcomes of a computation from being inferred.

The recent progress in human genome research [31,36] has made a great demand on convenient access to sensitive human genome data for research purpose. The problem of balancing privacy protection and data sharing in this domain, however, has not been seriously studied until Homer, et al. published their findings [39] a couple years ago. After that, several research groups, including us, have started working on this important issue [52, 46, 40, 51, 21]. As a prominent example, Sankararaman, et al [46] recently propose a technique (SecureGenome) for measuring the maximum statistical powers achievable on a set of single-allele frequencies. Most of these studies focus on single allele frequencies, which has been found in prior research to be insufficient [52], as sensitive information can also be inferred from other sources like test statistics. The research presented in this paper is the first attempt to understand and assess the risk in releasing different types of aggregate data, under typical inference threats.

Recovering SNP sequences is related to the research on contingency table release [20. 38, 23, 54, 27], and discrete tomography [37], which tries to reconstruct a matrix from a small number of projections. However, the specific problem of restoring a matrix from pair-wise allele counts is new, up to our knowledge, and the related complexity problems have not been studied before.

The Red-zone data identified by our techniques are not supposed to be released directly. However, they could still be published after proper sanitization and obfuscation. Such techniques have been studied in data-based privacy [17, 30, 19]. Particularly, the privacy policy based upon Differential privacy [29], once enforced, can make an identification impossible. Therefore, an important research direction is to develop effective techniques to achieve such a privacy objective on aggregate human genome data. 


\section{Conclusion}

Availability of aggregate human DNA data is of great importance to human genome studies. Recent research shows that such data are vulnerable to different types of privacy threats, which could lead to identification of the participants of these studies and disclosure of their sensitive genetic markers. Therefore, a critical question becomes how to evaluate such a risk and determine when the data are safe to release. In our research, we make the first attempt to answer this question. We identified the problem space of aggregate data release, considering both different types of data available in the public domains (allele frequencies and test statistics) and common threats to such data (identification attack and recovery attack). Through a systematic exploration of the space, we gained an important new understanding of the problem. Specifically, we found that inferring useful information from such data is difficult in general: the adversary often does not have enough information and needs to solve NP-complete or NP-hard problems. On the other hand, we also show that an attack can still happen under some circumstances, particularly when the solution space of the problem is small. Based upon such an understanding, we propose a new risk-scale system that determines when data can be safely released, through analyzing their solution spaces.

Given the scale and the depth of this data-release problem, many open issues remain in the problem space. Particularly, a critical issue here is how to narrow the range of the Yellow zone, to get tighter bounds for releasing or not releasing an aggregate dataset. Also important is the study on new anonymization techniques that obfuscate the Red-zone data to achieve differential privacy without substantially compromising their scientific value.

\section{References}

1. Haplotype Estimation and Association (2005), http://slack.ser.man.ac.uk/ theory/association_hap.html

2. NIH Background Fact Sheet on GWAS Policy Update (2008), http://grants .nih. gov/grants/gwas/background_fact_sheet_20080828.pdf

3. fastPHASE (2010), http: / / stephenslab.uchicago.edu / sof tware.html

4. Genome-Wide Association Studies (2010), http://grants.nih.gov/grants/ gwas /

5. Ibm ilog cplex optimizer (2010), http://www-01.ibm.com/software/ integration/optimization/cplex-optimizer/

6. International HapMap Project (2010), http : / /www . hapmap . org /

7. National Institutes of Health (2010), http: / / www.nih.gov/

8. Policy for sharing of data obtained in nih supported or conducted genome-wide association studies (gwas) (2010), http://grants.nih.gov/grants/guide/ notice-files/not-od-07-088.html

9. The R project for statistical computing (2010), http: / /www.r-project.org/

10. Re-identification and its discontents (2010), http://www . genomicslawreport.com/index.php/2009/10/13/ re-identification-and-its-discontents/

11. SecureGenome (2010), http://securegenome.icsi.berkeley.edu/ securegenome/

12. SNP.plotter (2010), http://cbdb.nimh.nih.gov/ kristin/snp.plotter. html 
13. Wellcome Trust Case Control Consortium (WTCCC1) (2010), https : / /www . wt cCC . org.uk/ $\mathrm{CCC} 1 /$

14. Agrawal, D., Aggarwal, C.C.: On the design and quantification of privacy preserving data mining algorithms. In: PODS 2001: Proceedings of the twentieth ACM SIGMOD-SIGACTSIGART Symposium on Principles of Database Systems, pp. 247-255. ACM, New York (2001)

15. Agrawal, R., Srikant, R.: Privacy-preserving data mining. SIGMOD Rec. 29(2), 439-450 (2000)

16. Atallah, M.J., Kerschbaum, F., Du, W.: Secure and private sequence comparisons. In: WPES 20: Proceedings of the 2003 ACM Workshop on Privacy in the Electronic Society, pp. 3944. ACM, New York (2003)

17. Barak, B., Chaudhuri, K., Dwork, C., Kale, S., McSherry, F., Talwar, K.: Privacy, accuracy, and consistency too: a holistic solution to contingency table release. In: PODS 2007: Proceedings of the Twenty-sixth ACM SIGMOD-SIGACT-SIGART Symposium on Principles of Database Systems, pp. 273-282. ACM, New York (2007)

18. Beck, L.L.: A security machanism for statistical database. ACM Trans. Database Syst. 5(3), 316-3338 (1980)

19. Blum, A., Dwork, C., McSherry, F., Nissim, K.: Practical privacy: the sulq framework. In: PODS 2005: Proceedings of the Twenty-fourth ACM SIGMOD-SIGACT-SIGART Symposium on Principles of Database Systems, pp. 128-138. ACM, New York (2005)

20. Bonferroni, C.E.: Teoria statistica delle classi e calcolo delle probability. Pubblicazioni del R Istituto Superiore di Scienze Economiche e Commerciali di Firenze 8(1), 3-62 (1936)

21. Braun, R., Rowe, W., Schaefer, C., Zhang, J., Buetow, K.: Needles in the haystack: Identifying individuals present in pooled genomic data. PLoS Genet 5(10), e1000668 (2009)

22. Bruekers, F., Katzenbeisser, S., Kursawe, K., Tuyls, P.: Privacy-preserving matching of dna profiles. Technical Report Report 2008/203, ACR Cryptology ePrint Archive (2008)

23. Chen, Y., Diaconis, P., Holmes, S.P., Liu, J.S.: Sequential monte carlo methods for statistical analysis of tables. Journal of the American Statistical Association 100, 109-120 (2003)

24. Chin, F.Y., Ozsoyoglu, G.: Auditing and inference control in statistical databases. IEEE Trans. Softw. Eng. 8(6), 574-582 (1982)

25. Chiò, A., Schymick, J.C., et al.: A two-stage genome-wide association study of sporadic amyotrophic lateral sclerosis. Hum. Mol. Genet. 18(8), 1524-1532 (2009)

26. Chvatal, V.: Recognizing intersection patterns. In: Combinatorics 79, Part I, pp. 249-251. North-Holland Publishing Company, Amsterdam (1980)

27. Dobra, A., Fienberg, S.E.: Bounds for cell entries in contingency tables induced by fixed marginal totals. Statistical Journal of the United Nations ECE 18, 363-371 (2001)

28. Duerr, R.H.H., et al.: A genome-wide association study identifies il23r as an inflammatory bowel disease gene. Science (October 2006)

29. Dwork, C.: Differential privacy. In: Bugliesi, M., Preneel, B., Sassone, V., Wegener, I. (eds.) ICALP 2006. LNCS, vol. 4052, pp. 1-12. Springer, Heidelberg (2006)

30. Dwork, C., McSherry, F., Nissim, K., Smith, A.: Calibrating noise to sensitivity in private data analysis. In: Halevi, S., Rabin, T. (eds.) TCC 2006. LNCS, vol. 3876, pp. 265-284. Springer, Heidelberg (2006)

31. Edwards, A.O., Ritter, R., et al.: Complement factor H polymorphism and age-related macular degeneration. Science 308(5720), 421-424 (2005)

32. Fienberg, S.E.: Datamining and disclosure limitation for categorical statistical databases. In: Proceedings of Workshop on Privacy and Security Aspects of Data Mining, Fourth IEEE International Conference on Data Mining (ICDM 2004), pp. 1-12. Nova Science Publishing, Bombay (2004)

33. Gehrke, J.: Models and methods for privacy-preserving data analysis and publishing. In: ICDE 2006: Proceedings of the 22nd International Conference on Data Engineering, p. 105. IEEE Computer Society, Washington, DC, USA (2006) 
34. Goldreich, O., Vadhan, S.: Special issue on worst-case versus average-case complexity editors' foreword. Comput. Complex. 16, 325-330 (2007)

35. Greenspan, G., Geiger, D.: Modeling haplotype block variation using markov chains. Genetics 172(4), 2583-2599 (2006)

36. Haines, J.L., et al.: Complement factor $\mathrm{H}$ variant increases the risk of age-related macular degeneration. Science 308(5720), 419-421 (2005)

37. Herman, G.T., Kuba, A.: Advances in Discrete Tomography and Its Applications (Applied and Numerical Harmonic Analysis). Birkhauser, Basel (2007)

38. Hoeffding, W.: Scale-invariant correlation theory. Masstabinvariante Korrelationstheorie, Schriften des Matematischen Instituts und des Instituts fr Angewandte Mathematik der University 5, 179-233 (1940)

39. Homer, N., et al.: Resolving individuals contributing trace amounts of dna to highly complex mixtures using high-density snp genotyping microarrays. PLoS Genet. 4(8), $\mathrm{e} 1000167+(2008)$

40. Jacobs, K.B., et al.: A new statistic and its power to infer membership in a genome-wide association study using genotype frequencies. Nature Genetics 41(11), 1253-1257 (2009)

41. Jha, S., Kruger, L., Shmatikov, V.: Towards practical privacy for genomic computation. In: 2008 IEEE Symposium on Security and Privacy (2008)

42. Kim, Y., Feng, S., Zeng, Z.B.: Measuring and partitioning the high-order linkage disequilibrium by multiple order markov chains. Genet. Epidemiol. 32(4), 301-312 (2008)

43. Morris, A.P., Whittaker, J.C., Balding, D.J.: Little loss of information due to unknown phase for fine-scale linkage-disequilibrium mapping with single-nucleotide-polymorphism genotype data. Am. J. Hum. Genet. 74(5), 945-953 (2004)

44. Renström, F., et al.: Replication and extension of genome-wide association study results for obesity in 4,923 adults from northern sweden. Hum. Mol. Genet. (January 2009)

45. Robbins, R.: Some applications of mathematics to breeding problems iii. Genetics 3(4), 375-389 (1918)

46. Sankararaman, S., Obozinski, G., Jordan, M.I., Halperin, E.: Genomic privacy and limits of individual detection in a pool. Nat. Genet. 41(9), 965-967 (2009)

47. Scott, L., et al.: A genome-wide association study of type 2 diabetes in finns detects multiple susceptibility variants. Science (April 2007)

48. Sladek, R., et al.: A genome-wide association study identifies novel risk loci for type 2 diabetes. Nature (February 2007)

49. Stephens, M., Donnelly, P.: A comparison of bayesian methods for haplotype reconstruction from population genotype data. American Journal of Human Genetics 73(5), 1162-1169 (2003)

50. Stephens, M., Smith, N., Donnelly, P.: A new statistical method for haplotype reconstruction from population data. The American Journal of Human Genetics 68(4), 978-989 (2001)

51. Visscher, P.M., Hill, W.G.: The limits of individual identification from sample allele frequencies: Theory and statistical analysis. PLoS Genet. 5(10), e1000628 (2009)

52. Wang, R., Li, Y.F., Wang, X., Tang, H., Zhou, X.: Learning your identity and disease from research papers: Information leaks in genome wide association study. In: CCS 2009: Proceedings of the 15th ACM Conference on Computer and Communications Security, pp. 534-544. ACM, New York (2009)

53. Yeager, M., et al.: Genome-wide association study of prostate cancer identifies a second risk locus at 8q24. Nature Genetics 39(5), 645-649 (2007)

54. Yuguo Chen, I.H.D., Sullivant, S.: Sequential importance sampling for multiway tables. The Annals of Statistics 34(1), 523-545 (2006)

55. Zhou, X., Peng, B., Li, Y.F., Chen, Y., Tang, H., Wang, X.: Technical report tr696: To release or not to release: Evaluating information leaks in aggregate human-genome data (2011), https://www.cs.indiana.edu/cgi-bin/ techreports $/$ TRNNN. cgi ?trnum=TR696 


\section{A Terminologies}

Table 1. HGS Terminologies used in this paper

\begin{tabular}{|l|l|}
\hline Terminologies & Description \\
\hline Polymorphism & $\begin{array}{l}\text { The occurrence of two or more genetic forms (e.g. alleles of SNPs) among } \\
\text { individuals in the population of a species. }\end{array}$ \\
\hline $\begin{array}{l}\text { Single Nucleotide } \\
\text { Polymorphism } \\
\text { (SNP) }\end{array}$ & $\begin{array}{l}\text { The smallest possible polymorphism, which involves two types of nu- } \\
\text { cleotides out of four (A, T, C, G) at a single nucleotide site in the genome. }\end{array}$ \\
\hline Allele & $\begin{array}{l}\text { One of the two sets of DNAs in a human individual's genome. Most SNP } \\
\text { sites have two common alleles in the human population: the major allele } \\
\text { (denoted by 0), the one with higher frequency, and the minor allele (denoted } \\
\text { by 1), the one with lower frequency. }\end{array}$ \\
\hline Genotype & $\begin{array}{l}\text { The combination of two set of alleles in a human individual. For a SNP site } \\
\text { with two common alleles, there are three possible genotypes: two homozy- } \\
\text { gotes, 00 and 11, and one heterozygote 01. }\end{array}$ \\
\hline Locus(plural loci) & The surrounding regions of a SNP site in the genome . \\
\hline Haplotype & $\begin{array}{l}\text { Haplotype, also referred to as SNP sequence, is the specific combination } \\
\text { of alleles across multiple neighboring SNP sites in a locus. Each individual } \\
\text { has two haplotypes, each inherited from one parent. Some haplotypes are } \\
\text { more common than others in the population. }\end{array}$ \\
\hline $\begin{array}{l}\text { Linkage disequilib- } \\
\text { rium(LD) }\end{array}$ & \begin{tabular}{l} 
Non-random association of alleles among multiple neighboring SNP sites. \\
\hline
\end{tabular} \\
\hline
\end{tabular}

\section{B Proofs of Theorem 1, Corollary 1, Corollary 2 and Corollary 4 Conjecture 1, Theorem 2, Corollary 5, Corollary 6}

Proof of Theorem 1 This problem can be formalized as an existence problem $\operatorname{EP}\left(C_{i}, C_{i j}\right)$ which is to determine whether an $N \times L$ binary matrix $M$ exists that satisfies the constraints of the sets of single allele counts $\left\{C_{i \in[1, L]}\right\}$ (e.g. the number of 0 s in column $i$ ) and pairwise major allele counts $\left\{C_{i j \in[1, L]}\right\}$ (e.g. the number of 00 pairs of column $i$ and column $j$ ). NOTE that these two sets are equivalent to the set of pairwise allele frequencies and may be used interchangeably in this paper. e.g. $C_{i}=3$, termed as 3$\operatorname{EP}\left(C_{i}, C_{i j}\right)$. Consider a special case of $\operatorname{EP}\left(C_{i}, C_{i j}\right)$, denoted as $3-\mathrm{EP}$, where all given single allele counts are $3\left(C_{i}=3\right)$. We prove 3 -EP is NP-complete by reducing the 3-Recognizing Intersection Patterns Problem(3-RIPP $(A)$ ), a known NP-complete problem [26] to it. 3-RIPP $(A)$ is described as: given $A=\left[a_{i j}\right]_{L \times L}$ in which $a_{i i}=3$, is there an integer set collection $H=\left\{H_{1}, H_{2}, \cdots, H_{L}\right\}$ such that $a_{i j}=\left|H_{i} \cap H_{j}\right|$ for $1 \leq i, j \leq L$. Obviously, 3-EP $\in$ NP. Given an arbitrary instance of 3-RIPP $(A)$, we construct an instance of $3-\operatorname{EP}\left(C_{i}, C_{i j}\right)$ by setting $C_{i j}=a_{i j}$ for $1 \leq i \neq j \leq L$ and setting $C_{i}=a_{i i}$ for $1 \leq i \leq L$. Suppose $M_{N \times L}$ is a solution of EP. We can convert each column of $M_{N \times L}$ into a set, where the row indices of $1 \mathrm{~s}$ in the $i$-th column form the elements in the set $H_{i}$. Therefore, We get $\left|H_{i} \cap H_{j}\right|=a_{i j}=C_{i j}$ for $1 \leq i, j \leq$ $L, i \neq j$ and $\left|H_{i} \cap H_{i}\right|=a_{i i}=C_{i}=3$. So $\left\{H_{i}\right\}$ represent a solution of $3-\operatorname{RIPP}(A)$. Conversely, suppose $H=\left\{H_{1}, H_{2}, \cdots, H_{L}\right\}$ is a solution of $3-\operatorname{RIPP}(A)$. We can 
construct a solution $M$ of 3-EP by converting each set $H_{i}$ into a column of length $L$ where for each element $k \in H_{i}$, fill in the $k$-th position by 1 in the $i$-th column of $M_{N \times L}$, and all the other positions by 0 . Clearly the resulting matrix $M_{N \times L}$ is consistent with $\left(C_{i}, C_{i j}\right)$, and thus is a solution of $3-E P$. Because the conversions described above can be done in polynomial time, 3-EP $\left(C_{i}, C_{i j}\right)$ is NP-complete. Therefore, $\operatorname{EP}\left(C_{i}, C_{i j}\right)$ is also NP-complete since its special case $3-\operatorname{EP}\left(C_{i}, C_{i j}\right)$ is NP-complete.

The rest of the proof is given in our technique report [55]. 